\title{
A Universal Perspective On Communication: What Every International Business Manager Should Know
}

Donald C. Smith, (E-mail: DCSmith@ newhaven.edu), University of New Haven

\begin{abstract}
Communication is neither good nor bad. It is simply a tool. Used well it nets good results, applied poorly it makes situations worse. The key then, for any manager, is to properly assess a situation and subsequently choose an appropriate strategy for participation. Intercultural Communication events are especially challenging with so many variables at play. Drawing upon the concept of "The Expert Spectator" this paper demonstrates that a universal perspective of communication exists, one that can be applied regardless of cultural orientation. When communication is seen on a continuum from the expressive to the instrumental it is much easier to be an appropriate and effective communicator in International Business settings.
\end{abstract}

\section{COMMUNICATION IS NEITHER GOOD NOR BAD}

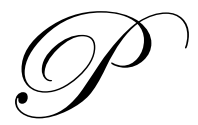

erhaps of all the myths that Richmond and McCroskey discuss the biggest of them is that "Communication is good." (Richmond and McCroskey, 2002). For many "communication" is simply a buzzword - when a problem is encountered participants announce, "we're not communicating," or "we need to communicate more." "Let's sit down and talk about it." Those trained in the field know that simply talking about something is not necessarily a good thing. Situations can become progressively worse when dysfunctional communication is at hand and if the discussants are not trained to recognize when such is the case, dialogue spirals in a downward direction. If a hammer is used to build a house this is a good thing. If it is used to assault a neighbor, this is bad. Similarly communication can create or destroy.

\section{EFFECTIVE COMMUNICATION}

Communication is a process of using symbols and referents wherein participants hope to arrive at a meeting of minds. The degree to which a sender's intent matches what a receiver understands is directly proportional to the level of success. When $\mathrm{S} / \mathrm{R}=1$, perfection has occurred. (Tubbs, 2000). Perfection, as we know, is rare.

\section{CULTURE DEFINED}

Culture itself is a communication construct. It can be defined as a way of life developed and handed down from generation to generation. (Dodd, 1995) Consider this: if an Irish infant from New England were somehow lost in the hills of China, separated from his or her parents, and there raised into adulthood; this child would, culturally be Chinese. Raised to speak Chinese, eat Chinese, engage in Chinese customs; for all intent and purposes this child who looks Irish is culturally Chinese. Thus it becomes important to consider not how a person looks or where they live but rather what have they learned and what sense of identity have they constructed.

Interestingly, culture exists for a person on at least two levels. First there is what someone understands about the norms, values, and attitudes of the culture as a whole - the macro level of understanding. Second is what an individual has internalized as their own core beliefs about a society's norms, values, and attitudes. Arguably no two individuals experience their cultural identity in exactly the same way. (Smith, 1999). As people are socialized into a 
system there are things they accept and things they reject. And, there is compliance for fear of negative social consequences, as well as those things genuinely accepted for personal reasons.

\section{WORLD VIEWS}

Each individual has different life experiences and there is a wide range of meaning that can be assigned to these events. In total these serve as the individual's world-view and as the foundation through which they screen communication. Is marriage good or bad? Which is more important job or family? What constitutes wealth? Which should occur first, a solid interpersonal relationship or the completion of a business specific task? Is fishing fun or boring? The list is endless and the point timeless.

As communicators we cannot just consider what we intend with a message, we must focus on what someone is doing with a message. Because so many variables are involved and because situations are even more complicated when cultural differences enter the picture, it is helpful to have a broad understanding of context in order to be at least nominally appropriate. The discussion below of "expressive" vs. "instrumental communication," will be of help in this regard. "Expressive communication," is communication for communication's sake - it has no purpose other than to share and where communicators find common ground, connections are made, trust grows, relationships evolve. At the other end of the continuum we have "instrumental communication," i.e. communication specifically designed to get a task accomplished. It is focused and goal oriented. While the lines of demarcation are not so clearly drawn in communication events, and while all communication can lie anywhere along this continuum, it is very important to ask, where along the lines of this model do participants seem to be operating from? Does the communication situation currently before me appear to an "expressive" opportunity or an "instrumental" one? Thinking along these lines allows a communicator to adapt appropriately and to consider how to take the conversation in what might be a more preferential or appropriate direction. As many management texts would suggest all business involves a concern for task and a concern for people. The model proposed in this paper empowers managers, at a glance, to consider which communication context, "expressive" or "instrumental" is primarily at hand. Once they become "Expert Spectators" in this regard, they can move on to becoming expert communicators. Let us turn then, to discussing the "Expert Spectator," and to completely outlining our model of "expressive" v. "instrumental" communication.

\section{THE EXPERT SPECTATOR}

The concept of the expert spectator and its relationship to management is succinctly explained in the following excerpt:

Expert training in a field allows people to see things they would not have noticed otherwise. In the hospital, a trained eye looks at an $x$-ray and sees much more than would the average citizen. An athlete turned television commentator observes the playing field and is able to discuss not just what has happened but what else might have happened and why. This person knows the players and the possibilities. A chorale director listening attentively to the hundred voices before him can stop and single out the one voice that is off key and make correction. In each instance it is the formal training that allows each of these people to see and hear what others could not.

Communication is a formal field, like medicine or law, and has a rich tradition of concepts and theories associated with it dating at least back as far as 5 BC. How is the concept of the "Expert Spectator," relevant here in regard to management and culture? Consider that,

Communication analysis, in the role of the "Expert Spectator", (a term coined by Rosenfield, 1968 and adapted for use in this paper) would allow managers to examine what they have intended in an organization in relation to what an employee has understood. This kind of manager would not only ask, what was I intending with my communication. Instead, trained in the field, they would ask, what is my employee doing with my communication? What meaning have they assigned? What is in the way of their understanding? What motivation do they have? Why? The smart manager knows that by observing his or her employees, and by asking the right questions, these answers can be achieved. Why would any manager not want to do this in the first place? (Smith, 2003) 
Why, indeed. Certainly most managers would want to adopt any position that would make them more effective in their roles. But, as stated earlier, desire is not enough. Wanting to become expert at medicine, law, chemistry, communication, any profession, requires training in the field. Practitioners need to know what to look for and how to do it.

\section{EXPRESSIVE V. INSTRUMENTAL COMMUNICATION}

The value of viewing communication along a continuum from the "expressive" to the "instrumental" is that the model alerts managers to at least one important level of communication exchanges. Also, it provides a perspective that can be applied to any communication situation, regardless of the language at play. This makes it ideal for summarizing intercultural communication contexts.

On the one end of our continuum we have expressive communication. Under such circumstances communicators speak their minds with the goal of being understood. Journal writing would be one example in this regard. Poetry and music two others. In a CBS 60 minutes interview Bob Dylan was asked what he thought of being the voice of a generation. Did he know that "Blowing in the Wind" was an anthem for the sixties? Dylan's response was, "hey man, I was just trying to write songs." Dylan didn't think of himself in those terms. Arguably he was just trying to expressive himself at a particular point and time. In expressive communication settings we generally seek to know ourselves or share ourselves with other people. This type of communication is especially successful at forging bonds between and among people as common ground is found and trust grows. The climate in these situations is supportive. An individual feels free to speak as they sense no harm will come to them from doing so. The focus is on the person or people. Dialogue proceeds from the perspective of multiple realities. There is no one right answer. Difference of opinion is good, indeed anticipated. The dialogue is free form. In the end people know themselves and others better. We have collaboration and cooperation.

On the far other end of the continuum is instrumental communication. In this context communicators speak with the goal of achieving a specific objective. The climate is competitive and possibly combative. There are right answers and these must be found and supported. There is a job to be accomplished and all thought must be focused along these lines. When taxes have to be filed by April $15^{\text {th }}$, there are no multiple realities, that indeed is the deadline, and if unmet real consequences follow assuming no extension is requested. As the space shuttle returns to earth there are limited windows of opportunity for reentry and limited landing spaces. When heart failure occurs minutes count. Medical professionals need to move quickly. In law, once a person has been put to trial once, they cannot be put through the process twice for the same crime. Communication under these circumstances is much different when compared with expressive communication, and, for obvious reasons the focus is predominantly on task as opposed to people.

In the role of an "expert spectator" and viewing communication as a continuum along the lines of "expressive" v. "instrumental" is helpful to managers as it supplements what they may already know about people v. task orientation. Generally it is argued that when a manager has achieved a balance between concern for people and concern for task, this is ideal. From a communication perspective, however, such is less true. Certain circumstances require taking a stance from one position or the other. Humming, for example would be oriented to the left of the continuum, there really are no hard and fast rules for this form of communication. Communication oriented around brain surgery, however, would be organized around the far right. Communication styles of surgeons are the stuff of legends. Still many fail to realize that the professional's style is related to the objective they face. If a vessel starts to bleed and the patient is on the road to "crashing", there's not a whole lot of time to stop and weigh in on how everyone is feeling at the moment.

\section{IMPLICATIONS FOR INTERNATIONAL BUSINESS}

There's a terrific story of President Carter having traveled to Japan. He began his remarks to the audience by telling a joke in English. The translator did his job and the joke was a huge success. Later in the day the President asked the translator, "what exactly did you say in the translation"? At first he hesitated but when pressed admitted 
that the translation amounted to: "Ladies and Gentlemen, the President of the United States has just told a joke, please laugh." Clearly this individual knew how to adapt to a circumstance.

When involved in international business situations, communicators can similarly and quickly orient themselves by asking broadly - what type of situation is this before me? Is it one that provides primarily an expressive opportunity or one calling for instrumental skills? It should be noted that communication situations are not linear but organic and may shift constantly from one direction to the next. A manager should be aware of these shifts. Often, based upon custom, ritual, or circumstance the communication event will require a substantial orientation to the right or left of the continuum. Cognizant of this fact the manager is always set to engage in the most appropriate of communication behaviors and best positioned to take the communication in the direction he or she chooses if deemed prudent. The level of challenge can be significant:

Managers in multinational corporations encounter individuals with word-views substantially different than their own. Consider the following example: 'Returning to work after the death of a parent, one Euro-American employee appreciated and valued all of the co-workers who expressed sympathy and asked questions about the deceased parent. Someone of the Dine (Navajo) background could be extremely uncomfortable with the mention of the person who died and discussions of death, burial, and the life of the deceased. Because of cultural taboos, such conversations might produce greater anxiety instead of comfort.' (Smith, 2003, Adler, 2002 pp 51-52)

Daily, communicators in international business face a whole array of differences: customs, work habits, the list is extensive:

Differences in corporate interpretations, individually and collectively, might revolve around task attractiveness, social style appearance, punctuality, family obligations, civility, motivation, gender, contractual obligations, hierarchy, and overall conditions of employment. The language a manger uses has to be carefully considered. 'Mission' has a very different meaning in Southeast Asia than it does in the United States. (Smith 2003)

Due to the complexity it is very difficult to respond to every variable encountered. It is both possible and useful however to have a perspective that allows quick and broad orientation to international business communication situations. Regardless of the number of variations in a setting, always the event will be primarily people oriented or task orientated and the appropriate communication form will be expressive or instrumental.

\section{CONCLUSION}

The goal of this paper has been to provide a universal perspective on communication and to empower international business managers with insight they previously may have lacked. While the territory of intercultural communication is tricky and laden with pitfalls, viewing communication broadly along the lines of the continuum discussed in this work should consistently provide managers a vantage point for appropriate entry into a limitless number of communication encounters.

\section{REFERENCES}

1. Adler, R. B. Communication at Work. New York: Mc-Graw-Hill Higher Education.

2. Dodd, C. (1995). Dynamics of Intercultural Communication.

3. Richmond, V. (2002). Organizational Communication for Survival. Boston: Allyn and Bacon.

4. Smith, D. C. (2002). Culture and Identity: Your Right to Define? Speech Communication Association of Puerto Rico Convention, San Juan, December 1999.

5. Smith, D. C. (2003). Communication, Management, and the Role of The Expert Spectator: Adapting to cocultures in the workplace. Australia New Zealand Communication Conference, Brisbane, July 2003.

6. Tubbs, S. L. (2000). Human Communication. New York: McGraw-Hill Higher Education.

7. Tubbs, S. L. (2003). A Systems Approach to Small Group Interaction. McGraw-Hill. 